This is the pre-peer reviewed version of the article 'The erotetic theory of attention: Questions, focus, and distraction' by Philipp Koralus which has been reviewed and is forthcoming with Mind \& Language.

\title{
The Erotetic Theory of Attention: Questions, Focus, and Distraction
}

\author{
PHILIPP KORALUS \\ Forthcoming in Mind \& Language \\ $9 / 23 / 12$
}

\begin{abstract}
Attention has a role in much of perception, thought, and action. On the erotetic theory, the functional role of attention is a matter of the relationship between questions and what counts as answers to those questions. Questions encode the completion conditions of tasks for cognitive control purposes, and degrees of attention are degrees of sensitivity to the occurrence of answers. Questions and answers are representational contents given precise characterizations using tools from formal semantics, though attention does not depend on language. The erotetic theory proposes an integrated account of attention in cognitive control and of attentional focus in perception. The functional role of attentional focus on objects, properties, and locations has to do with picking out something that corresponds to what a task is 'about'. The erotetic theory of attention opens new avenues in theorizing about the relationship between attention, representational content, phenomenal character, and practical reason. A novel representationalist account of salience is proposed. The theory also provides an account of distraction that suggests when distraction is a defect in practical reasoning.
\end{abstract}

\section{Introduction}

Attention has a role in much of perception, thought, and action. Recent years have seen arguments to suggest that attention sheds light on a wide range of topics in mind and language (Block 2010, Prinz 2011, Elian et al. 2005, Campbell 2002, inter alia). This makes puzzling the paucity of general accounts of attention. Few approaches match Mole

For comments and discussions, I thank John Doris, Aaron Schurger, Wayne Wu, Susanna Siegel, Dave Chalmers, Evan Thompson, Bence Nanay, Todd Braver, and an anonymous reviewer. The work was supported by a fellowship at the Notre Dame Institute for Advanced Study.

Address for correspondence: Washington University in St. Louis, Philosophy-NeurosciencePsychology Program, One Brookings Drive, St. Louis, MO 63130, USA

Email: pkoralus@alumni.princeton.edu 
(2011) in attempting to characterize the functional role of attention across all cognitive domains. Roughly, attention, according to Mole, consists in the unity of cognitive processes dedicated to an agent's task. ${ }^{1}$

The cognitive unison theory makes attention an exclusive and ungraded all-ornothing phenomenon (prima facie cases of partial distraction have to be explained away), which not everyone will find to be an attractive consequence. Moreover, many philosophical questions about attention are centrally concerned with the contribution of attention to phenomenal character and content. The cognitive unison theory sheds little light on this contribution. Finally, more work needs to be done to make sense of distraction, the 'flipside' of attention. Considerations like these suggest that it is worth putting an alternative view on the table and exploring its consequences.

On the erotetic theory of attention proposed in this paper, the functional role of attention is a matter of the relationship between questions and what counts as answers to those questions. Questions encode the completion conditions of tasks for cognitive control purposes, and degrees of attention are degrees of sensitivity to the occurrence of answers. The functional role of attentional focus on objects, properties, and locations is accounted for in terms of a role in the conditions for attention to tasks, having to do with what a task is 'about'. What this comes to as a general theory of attention, and why it does not bar non-linguistic creatures from attention, will become clear in due course.

I will put to new use insights from the formal semantics of questions to make sense of attention (Hamblin 1958; Groenendijk and Stokhof 1984; Krifka 2001). In essence, questions are structured sets of alternatives, in the domain of representational contents, rather than linguistic items. To draw a parallel with something more familiar, probability distributions similarly are structured sets of alternatives, though they involve a different and much more complex kind of structure. Probability-based models of

\footnotetext{
${ }^{1} \mathrm{Wu}(2011)$ offers an independent view of attention in selection for action that shares important features with Mole's (with precursors in Allport 1987) and can be seen as describing the processes leading up to cognitive unison (Wu, p.c.). My primary aim is to present a new theory, and I will rely on Mole (2011) for contrast purposes. I do not have the space to discuss restricted approaches centered on attention and consciousness (e.g. Watzl 2011; Smithies 2011). As far as I can see, all of the above views are compatible with current proposals on neural mechanisms of aspects of attention like selection (Desimone and Duncan 1995; Reynolds and Heeger 2009), topdown influence (Kastner and Ungerleider 2000) and control (Botvinick et al. 2001). The ultimate aim should be integration of a high-level functional perspective, which bears most readily on philosophical issues, and a neural implementational perspective.
} 
aspects of the functional role of attention are fairly standard (e.g., Itti and Baldi 2005; 2009) and a model based on questions should be no more mysterious in principle.

The plan of the paper is as follows. First, I retrace arguments due to Mole (2011) that attention in its general form should be understood as a relationship between tasks and cognitive resources one may use to perform them. I will then present the erotetic theory of attention as an account of this relationship. Among other issues, I will discuss the possibility of divided attention, how we should think of aspects of the contribution of attention to perceptual phenomenal character, and varieties of distraction.

\section{Theory Types and the Centrality of Tasks}

At first blush, one might think that it is a purely empirical project for experimental psychologists to determine the nature of attention. On closer examination, some basic questions about the object of inquiry have to be answered at least in conjunction with empirical inquiry, if not in advance of it. One such question is whether attention is really a type of psychological process, or whether it is a manner in which psychological processes may carry on (see below for illustrations of the difference). Another question is what attention applies to in the broadest sense. I will retrace arguments due to Mole (2011) that we should think of attention as concerning the manner in which psychological processes carry on, and that attention applies to tasks.

\subsection{Process-Type and Process-Manner Theories}

Reconstructing an analogy due to Mole (2011), let us set aside attention and its underlying processes for the moment and imagine a group of Martians in the business of giving an account of the nature of employment and its underlying processes. Suppose a Martian goes to a factory to look for empirical data on the nature of employment and concludes that the underlying process of employment is the set of processes for arm and hand movements. Another Martian looks at a sports commentator and concludes that the underlying processes of employment are the processes for perception and speech. Brought together, the Martians might settle for a disjunctive theory of the underlying processes of employment: the processes of employment can either be the processes for arm and hand movement or the processes for perception and speech. Martian Science publishes their finding that employment is multiply realizable. 
Why does the Martian's conclusion seem wrongheaded? We would not take it that a disjunctive Martian theory of employment is wrong because we have not included enough disjuncts, or because the characterizations of the individual disjuncts might lack detail; we would take it that the Martians are mistaken about what sort of thing employment is in a way that cannot be fixed by just adducing more observations of the same sort. We want to say that the nature of employment is in the manner in which an activity is carried out, specifically, how it relates to reasons for action, etc.; the nature of employment is just not a type of activity (not even a disjunctive one). One might say that employment is not a process-type phenomenon, but a process-manner (or in Mole's terms, 'adverbial') phenomenon.

Return to the project of accounting for the nature of attention. Mole argues that cognitive psychology has tended to produce process-type theories of attention. For example, Treisman's feature integration theory of attention (Treisman and Gelade 1980; Treisman 1998) takes it that for the purposes of vision, attention just is the process that binds visual features into an object file. What this means is that pre-attentive stages in visual processing create representations of properties like colors or shapes, and attention is the process that binds those representations to a representation of an object. On this sort of view, attention would plausibly turn out to be a disjunctive process type, corresponding to the process of feature binding in the case of vision, and possibly to other processes in other domains.

According to a different sort of theory, attending to $X$ rather than $Y$ means that neural networks corresponding to $X$ have come out dominant in a certain type of competition. Attentional effort toward $X$ would mean straining to bias the competition in favor of $X$. This roughly describes the biased-competition model of attention (Reynolds and Desimone 2000; Desimone 2009). Here, attention is not identified with any particular process but with a manner in which those processes are executed, namely dominantly, in some relevant sense. Mole (2011) points to the biased-competition model as an example of a process-manner theory of attention, or an 'adverbial' theory, as Mole puts it.

In sum, Mole identifies two different sorts of theories of attention: On the one hand, we have process-type theories on which a particular process (more likely, a disjunctive range of processes) constitutes attention. On the other hand, we have processmanner theories, on which attention consists in a certain way in which these processes 
carry on, which could depend on how various processes relate to each other. On a process-type view, if one of the processes from the disjunctive range of processes that can constitute attention is active, then it must be sufficient for attention. On a processmanner view, no particular range of processes is necessary or sufficient for attention. Mole argues that no particular type of process is always sufficient for attention and concludes that a process-manner theory of attention is needed.

\subsection{The Task-Switching Argument and the Centrality of Tasks}

Consider two tasks: One task is to produce a string of random numbers. The other task is to calculate a complicated numerical series. Generating random numbers is very attention demanding if done at a quick pace (Jahanshahi et al. 2006), and the same is clearly true of calculating complicated numerical series. Mole (2011) points out that in order to attentively generate random numbers, it is not enough to just say whatever numbers happen to come to mind, since those tend to follow obvious patterns. Instead, attentively generating random numbers seems to involve rapidly switching between strategies for generating numbers to avoid creating patterns as much as possible. Getting stuck in one strategy would mean ceasing to attend to the task of generating random numbers. By contrast, attentively following the task of calculating a certain numerical series requires sticking with a certain strategy of generating numbers. Diverging from that strategy means ceasing to attentively follow the calculation.

Now, suppose you observe someone who first has an active psychological process $A$ corresponding to one strategy of generating numbers and then switches to a psychological process $B$ corresponding to another strategy of generating numbers. If that person is following the task of generating random numbers, then the observed switch could well be indicative of the fact that she is following her task attentively (by the considerations from the previous paragraph). By contrast, if that person is following the task of calculating a certain numerical series, then the switch between strategies could well be indicative of the fact that her attention is slipping. Mole argues that it is very plausible that we have pairs of tasks of this sort, where a switch from a psychological process $A$ to a process $B$ corresponds to a lack of attention if we are pursuing one task, but corresponds to full attention, if we are pursuing another task. If we have such pairs of tasks, then attention is not a matter of what type of psychological processes are active. 
Mole concludes that attention is not a matter of whether a particular kind of psychological process is active, but of how the activity of psychological processes relates to a task. The object of attention in its most general form is a task, broadly conceived, like calculating a sum, finding something to eat, etc., and we are performing a task attentively if the right kind of relation holds between the task and various psychological processes. We then have to say what this relation consists in.

In the following sections, I will give a novel account of the relation that has to hold between a task and various psychological processes in order for an agent to count as performing a task attentively: the erotetic theory of attention. I will later briefly present Mole's own account of this relation to contrast certain features of my own.

\section{The Erotetic Theory of Attention}

To begin with a rough preview, on the erotetic theory of attention, a task that we may follow attentively determines a question that encodes completion conditions for the task for the purposes of internal monitoring. Whether we are in fact following a task attentively depends on whether we are appropriately sensitive to the occurrence of answers in our cognitive economy. To flesh this out into a theory, it will be crucial to begin by developing a precise notion of questions and answers for the purpose at hand.

\subsection{Focus and the Semantics of Questions}

The term 'question', as I am using it in this paper, denotes something in the domain of contents. Interrogative sentences characteristically carry questions as their contents. For example, 'wer raucht?' ‘quien fuma?' and 'who smokes' semantically contribute the same question, though they are different interrogative sentences.

Hamblin (1958) proposed that while propositions determine truth conditions, questions determine answerhood conditions - what it takes to count as an answer. On any contemporary account in semantics, a question determines what counts as an answer to it. One can thus think of a question as determining a set of alternative answers. For example, where ' $\llbracket X \rrbracket$ ' denotes the semantic contribution of $X$, which is a question if $X$ is an interrogative sentence: 
$\llbracket$ Who smokes?》 $\rightarrow\{\text { Jack smokes, Sarah smokes, ..., Jack and Sarah smoke, ... }\}^{2}$

More recently, it has become clear that a complete account of answerhood has to go beyond the relationship of an answer to a set of alternatives determined by a question. In order to determine whether something counts as what has been called a congruent answer, additional considerations have to be taken into account. Focus marking in the answer has to correspond to the question in the right sort of way. In English, focus marking is generally indicated with contrastive stress. Other languages like Japanese have special particles for this purpose. To illustrate congruency, contrast the degree to which (6) and (7) count as acceptable answers to (5):

Q: Who smokes?
A: [Foc John] smokes.

A: *John [Foc $s m o k e s]$.

Relative to (5), (7) is not an acceptable answer. One could say that an answer has to match what a question is in some sense 'about'. Loosely, the question in (5) is about those who smoke and (6) but not (7) corresponds to this fact in the right way.

To capture how focus is involved in answerhood, Krifka (2001) proposes an analysis of questions in terms of a pair consisting of a function and a domain. The example question (5) would be analyzed as (8):

$$
\llbracket \text { Who smokes? } \rrbracket=<\lambda x \text {.Smoke }(x),\{y: \operatorname{Person}(y)\}>
$$

The function of the question in (8) (i.e. the left element in the pair on the right-hand side of the equation) takes us from an individual to the proposition that that individual smokes. For example, applying $\lambda x \cdot \operatorname{Smoke}(x)$ to John yields the proposition that John smokes. The domain of the question in (8) is the set of persons (which we may have to take to include groups). If we want to obtain the set of alternative answers of the

\footnotetext{
${ }^{2}$ For expository simplicity, I set aside the issue of whether sets of alternative answers should be thought of as partitions (imagine 'only' appended to every alternative) or whether they are merely covers (i.e. the alternatives are not exclusive). For a classic discussion, see Groenendijk and Stokhof (1984).
} 
question, we simply take the set of all those propositions we can obtain by applying the function to elements of the domain.

With this analysis of questions, we can capture the contrast between (6) and (7) as putative answers to (5): we say that a congruent answer to a question must have focus marking that corresponds to the domain of the question, besides supplying an alternative answer of the question. In (6), focus marking picks out John, who is indeed in the domain of the question (the set of persons). Moreover, the proposition that John smokes is indeed one of the alternative answers of the question. By contrast, in (7), focus marking picks out the property of smoking, which is not in the domain of the question. Thus, (7) is not a congruent answer to (5), but (6) is. We can then state the conditions for congruent answerhood in an integrated fashion as follows:

(Congruent Answerhood) Relative to a question $\langle F, D\rangle$ with a function $F$ and a domain $D$, a statement $S$ is a congruent answer if and only if what is focused in the statement picks out an element of $D$, and $S$ encodes the proposition one obtains by applying $F$ to what is focused in $S$.

So, more formally, '[Foc John] smokes' is a congruent answer to the question $<\lambda x$.Smoke $(x),\{y$ : Person $(y)\}>$, since John is an element of $\{y: \operatorname{Person}(y)\}$ and ' $\left[{ }_{\text {Foc }}\right.$ John] smokes' encodes the proposition one obtains by applying $\lambda x \cdot \operatorname{Smoke}(x)$ to John. ${ }^{3}$

We now have the following semantic machinery in place: questions are contents (not linguistic items) that consist in pairs of a function and a domain. In order for something to count as a congruent answer to a question, it must involve focus marking that picks out something in the domain of the question and it must encode one of the alternative answers determined by the question.

There should be nothing especially mysterious about the possibility of representing these contents non-linguistically. As argued by Burge (2009), the burden of proof is on those who would claim that non-linguistic cognitive states cannot bear

\footnotetext{
${ }^{3}$ An anonymous reviewer alerted me to the need to say something about acceptable answers like 'everyone smokes' to 'who smokes?' A standard move would be to say that the domain of the question includes not only individuals but also groups or pluralities, including the group of everyone. This would make 'everyone smokes' a straightforward alternative answer. A fuller discussion would go beyond what is strictly necessary for the basic picture about the role of questions in attention that I will propose.
} 
proposition-like content. If we allow for perceptual and other cognitive states to have proposition-like content, we should also allow questions as contents of such states. Fundamentally, questions correspond to structured sets of propositions, so they are not obviously more problematic. One could also observe that there already exist highly influential models of aspects of attention based on probability distributions in vision science (Itti and Baldi 2005; 2009). Probability distributions are themselves highly structured sets of alternatives, so the less complexly structured sets of alternatives that correspond to questions should be no more problematic.

A crucial part of the theory of attention proposed in this paper is that 'focus', for the purposes of attention, plays the same functional role focus marking plays in language with respect to answerhood conditions for questions. Thus, focus is not the same as attention but merely part of the conditions that determine whether something counts as an answer to a question. I will presently motivate why it makes sense to think of tasks as determining questions and why it makes sense to distinguish focus from attention.

Though nothing in the following will hinge on it, certain descriptions of the phenomenology of attention provide a useful though optional preliminary motivation for the basic picture of the erotetic theory of attention. Consider the below phenomenological characterization that owes one part to Mill and one part to Husserl:

$(\mathrm{M}+\mathrm{H})$ Attention involves distinguishing something 'by a mark' in perception (Mill 1865/1979, Ch. XVII) and attention presupposes an intention that 'strives after satisfaction' (Husserl, Hua X, pp. 146-152 cited in Dwyer, D. 2007).

If one takes it that the above characterizes an aspect of the phenomenology of attention, one can begin to sketch an account of it in terms of the erotetic theory of attention:

(9) Attention presupposes an intention that strives toward satisfaction in the sense that attention presupposes a question (determined by a task) that we seek to answer.

(10) Attention-related cognition involves distinguishing something by a mark in the sense that it involves focus marking that has a function analogous to focus marking in 
language with respect to determining what gets to count as a congruent answer to a given question.

I will now argue that it is useful to think of tasks as determining questions. Then, I will explain why it makes sense to think of focus as a cognitive feature closely related to- but distinct from attention.

\subsection{Tasks as Determining Questions}

We need to make sense of tasks for the purposes of developing a theory of attention. I will take the following starting point: every task (always understood as restricted to those for which the question can arise whether we are performing them attentively) aims at some range of goals that can be cognitively monitored (not necessarily consciously), and there is a fact of the matter whether various processes are cognizable as potentially contributing toward achieving the goal. We will need to unpack what is involved in monitoring task completion and what it takes for a cognitive process to be taken to have the potential to make a contribution toward a task goal.

It seems natural to suppose that every goal-directed task has to determine conditions under which a cognitive system can count it as completed. This does not mean that every task has an effectively possible completion, as in the task of staring at the wall at the present time instant and at every successive time instant. Across cognitive domains, imagining ourselves monitoring progress toward completing tasks, I suggest it is quite natural to think of task-completion conditions as monitored in the form of questions:

(Perception) Task of finding a banana $\rightarrow$ Where is a banana?

(Thought) Task of calculating the sum $2+2 \rightarrow$ What number is $2+2$ ?

(Action) Task of kicking the ball into the goal $\rightarrow$ Is the ball in the goal yet?

One of the reasons this move is natural is that normal task monitoring is intuitively about monitoring whether we are in some range of acceptable results. Thought of this way, the completion conditions of a task can be cashed out in terms of the answerhood conditions of a question that has the range of alternative acceptable results as its alternative answers. If I have an answer to the question 'where is a banana?' I have completed the task of 
finding a banana. Conversely, if I have found a banana, I have an answer to the question, 'where is a banana?" Similarly, if I have an answer to the question "what number is $2+2$ ?' I have completed the task of calculating the sum $2+2$.

There is evidence to suggest that for action tasks, the relevant kind of monitoring involved in proper performance is directed at the goal of the action, rather than at one's bodily states (McNevin et al. 2003; Wulf and Prinz 2001). Thus, it would make sense to think of the completion conditions for an action task in terms of a question about the target state of the world as well. Now, if we were to think of the question encoding the completion conditions for the task of kicking the ball into the goal along the lines of the interrogative 'is the ball in the goal?' in English we would face the problem that 'no' counts as an answer. This would be inappropriate, since the task cannot be completed by not putting the ball in the goal (you might be able to abandon the task that way). Fortunately, we now have an abstract formal framework for formulating questions with more flexibility and we were only interested in English to motivate the basic elements of that framework. Indeed, the whole picture would be implausible if we had to think of task monitoring as involving interrogative sentences in some natural language. We can construct appropriate questions directly in the formalism and drop the use of ordinary language approximations:

[Task of finding a banana]

[Task of calculating the sum 2+2]

(A)
[Task of kicking ball into goal]

$$
\begin{aligned}
& <\lambda x \text {.Banana_is_at_x, }\left\{y: y \_ \text {is_a_place }\right\}> \\
& <\lambda x \cdot 2+2=x,\left\{y: y \_ \text {is_a_number }\right\}> \\
& <\lambda x \text {.The_ball_is_in_x, }\{y: y \text { is_the_goal }\}>
\end{aligned}
$$

Ignore the issue of focus in determining answerhood conditions for the moment. Then, for any given place $p,(\mathrm{P})$ admits the proposition that a banana is at $p$ as an answer. ${ }^{5}$ For

\footnotetext{
${ }^{4}$ Once we make our questions for task-monitoring purposes precise, it becomes clear that tasks are more fine-grained than may be obvious at first. 'Where is a banana?' only suffices as a question for monitoring a very bare task of finding a banana, in the sense of a task of finding relief from lack of banana-location knowledge. This has to be distinguished from the task of finding one individual banana that is then somehow selected for further action. Suppose you learn the universal fact that bananas are strictly everywhere. This would conclude the bare task of finding relief from lack of banana-location knowledge. However, this would clearly not conclude the task of finding an individual banana and selecting it, or the task of finding a banana and giving one some further treatment.
} 
any number $n,(\mathrm{~T})$ admits the proposition that $n=2+2$ as an answer. In the case of (A), for any $g$ such that $g$ is the goal, the proposition that the ball is in $g$ counts as an answer. Still setting aside the role of focus, if the cognitive system can produce representations of relevant propositions, recognizing that a target state has been achieved, the tasks can be cognized as completed.

With the above, we have accounted for completion conditions for tasks for the purposes of monitoring in terms of answerhood conditions for questions determined by tasks. Of course, fulfilling the completion conditions for the purposes of monitoring does not guarantee that the tasks are completed by external standards. However, it would be absurd to demand that completion conditions for internal monitoring purposes be restrictive enough to enforce these standards. To make the point through an example, there is no task of calculating a sum left to be monitored, if the monitoring systems already know the right answer. This does not mean that external standards of success are irrelevant. Doing well at a task not only requires monitoring in the sense under discussion; it clearly also requires taking cognitive means to actually performing one's task that have a good chance of fulfilling external standards. However, what is involved in doing well at a task goes beyond what is involved in paying attention to a task.

By thinking of tasks as determining questions in the sense just discussed, we can not only provide an account of completion conditions for the purposes of monitoring tasks, but we can also give an account of how cognitive systems can determine what psychological processes count as making a direct contribution toward completing a task, or, put differently, what psychological processes match what a task is 'about'. Recall the task of calculating a certain sum. Suppose that while we are following this task, some psychological process that detects the presence of bananas in the visual field signals 'banana here!' Somehow, the very nature of the task of calculating a sum should rule out that 'banana here!' is a contribution. The representations involved in monitoring the task of calculating a sum should rule out that we could take the task as completed in virtue of the detection of a banana.

There is an important sense in which a process 'not being on task' is distinguishable from mere lack of success by some external standard; some psychological

\footnotetext{
${ }^{5}$ If we want to rule out that learning that there are bananas everywhere directly concludes our task, we could stipulate that our domain of places does not include everywhere, or does not include any sets of places.
} 
processes, as in the case at hand, do not even count as 'trying' to contribute. The task of calculating a sum is simply not about the same sort of thing as a banana detector. Contrast the forgoing scenario with a different one in which the psychological processes through which we carry digits in our calculations misfire through some fluke in neural processing. The number that these processes contribute in this case is not correct, but they contribute to the task of calculating a sum in a way that the banana-detecting processes do not. The number-producing psychological processes match what the task is about in way that the banana-detecting processes do not.

Since we have analyzed tasks as determining structured questions that encode completion conditions, we immediately get an account of the kind of match between tasks and cognitive resources just described. In order for a psychological process to potentially contribute to a given task, the following has to hold: it has to be possible for the contribution of that psychological process to match the domain of the question determined by the task and it has to be possible for the contribution of the psychological process to be a congruent answer to the question determined by the task. These considerations yield a general notion of task congruency of a psychological process relative to a task.

(Task congruency) A cognitive process $P$ is task congruent relative to a task $T$ if and only if there is a way of assigning focus to some $A$ in the range of possible outputs of $P$ such that $A$ is a congruent answer to $\left\langle F_{T}, D_{T}>\right.$.

For example, a psychological process that signals the presence of bananas is not going to be congruent to the task of calculating the sum $2+2$, since it has no hope of producing a congruent answer to the question 'what is $2+2$ ?' not even a false one.

In this section, I proposed that each task determines a question (in the nonlinguistic sense described) that cognitive systems can use to monitor task completion and whether the contribution of a psychological process is congruent to a task. Working toward completing a task involves working toward answering a question determined by the task.

We have considered examples of what these questions may look like for tasks in perception, thought, and action. It will not always be obvious what particular questions in 
fact correspond to what tasks. Yet, if we have a clear sense of the range of alternative states that would count as completions of the task, we can generate an appropriate question that has alternative answers corresponding to those states. It may seem surprising at first that all attentive tasks should involve a question, but it should be clear that this view is extremely flexible.

The approach sketched is neutral on whether there can be tasks with associated questions that are being followed sub-personally. At least some tasks are obviously conscious. Even if a task is conscious, we do not have to assume that the corresponding questions have to be introspectively accessible in a way that would make it intuitively obvious that we are dealing with questions. Recall the 'optional' Husserlian phenomenological description of attention as involving an intention that 'strives after satisfaction' (Husserl, Hua X, pp. 146-152 cited in Dwyer, D. 2007). If we think this description is correct, we can cash it out as follows: attention presupposes a task, which determines a question to be answered. The phenomenology of 'striving after satisfaction' in attention supervenes on the question that we seek to answer (which would mean completing our task). Alternatively, if we think Husserl is wrong, we could take it that our questions normally do not contribute to phenomenology.

For expository simplicity, I have avoided distinguishing between superordinate tasks and subtasks that are ways of pursuing aspects of the former. Though there is no space to pursue this issue here, the approach of monitoring tasks in terms of questions promises further advantages in this area, in the form of a precise account of the relationship between tasks and superordinate tasks. There are question logics that recognize that there are subquestions, answers to which serve as partial answers to broad questions (Groenendijk and Stokhof 1984). Thus, the erotetic theory of attention opens space to develop a formal account of the relationship between superordinate tasks (involving broad questions) and subtasks (involving subquestions). This will have to be left for future work.

\subsection{Distinguishing Focus and Attention}

I will now argue that once we think of attention as something that applies to tasks, it is desirable to have a notion of focus that is distinct from attention. 
Suppose we attentively follow the task of finding a red square in a display filled with red things of various shapes and squares of various colors, as in a typical visualsearch task in perceptual psychology (Treisman 1998; Treisman and Gelade 1980). Even though all objects in the display are impacting our sensory systems at once, participants will normally not detect the target immediately. On a standard model of this type of task, detecting a target defined by two features (color and shape in this case) in a sea of distractors sharing those features requires 'attentional focus' to pass over the target. Attentional focus cannot be applied to the whole display at once, so it has to be allocated to different parts in a serial fashion, predicting that search times increase steeply with the number of distractors. In normal visual search, the trajectory of attentional focus is not controlled by top-down conscious effort but is determined 'anarchically'. Search performance in fact slows down if we try to control the search path consciously (Wolfe 2000). Besides volition, particular tasks and expectations can clearly bias attentional focus. Yet, the processes that control attentional focus allocation in the normal course of events seem to be mainly driven by relatively low-level processing and the recent history of visual input, as suggested by the fact that computational models based on those variables yield surprisingly good predictions (Itti and Baldi 2005; 2009). I take it that focus in the sense under discussion is what can get captured in 'attentional capture'. What aspects of the visual scene are processed in a way that influences attentional focus continues to be under investigation (Jiang et al. 2006). There is also considerable debate about the nature and mechanisms of attentional focus (Wright and Ward 2008).

Now, if we want to say that attention is something we pay to a task, attentional focus of the kind just described is best not thought of as the same thing as attention, even if we limit our discussion to the visual domain. We can keep attentively following the task of finding the red square in the sea of distractors, while our focus moves about, from one potential target to the next. Our attention keeps its object, namely the task of finding the red square, while our focus jumps from one object to the next until we have completed the task or decided to abandon it. This makes it seem like attention in the sense under discussion in this paper is not the same as focus.

Similar examples can be found in other domains. Suppose we are attentively following the task of finding an incompetently played instrument in an orchestra. All of the instruments are sensorily impacting us at once. Our auditory focus can intuitively 
move about from one instrument to the next, while we keep attentively following the same task. Similar patterns seem possible in attentive thought.

Now, instead of admitting that attention in the sense at issue in this paper is distinct from focus, someone might argue that in the examples just discussed, our attention is shifting all the time between different sub-tasks, one for each movement of focus in search. However, I suggest that in a simple visual search task of the sort just considered, this makes tasks seem too fine-grained. As noted, where our focus goes from moment to moment in normal search seems largely determined by stimulus-driven bottom-up processing, which is not an obvious candidate for the attribution of tasks. It seems more natural to say that the bottom-up processing that produces focus can carry on largely independently, while task representations are involved in top-down processes that merely bias how otherwise anarchic focus is assigned. This would allow us to maintain that even if task monitoring may not always be conscious, relevant task representations most likely reside somewhere beyond sensory cortices. Thus, I suggest it is more attractive to keep separate notions of attention and focus.

I will take it that focus in the sense under discussion corresponds to what vision scientists tend to call 'attentional focus' or 'selective attention'. It amounts to a special way of marking certain positions or elements in a feature map, yielding all sorts of consequences for further processing. I will stay as neutral as possible on what could become marked by focus and simply speak of features and their combinations being focus marked. These could be perceptual or 'thought' features corresponding to properties like being red or having a certain shape, to objects and positions, and to combinations of objects and properties. For expository simplicity, I will refer to all those things as 'features'.

Now, on the erotetic theory of attention, at least one of the functional roles of attentional focus (which I will from now on just call 'focus') closely parallels that of focus in natural language with respect to answering questions. In order for a set of perceptual or thought features to count as an answer to the question determined by a task, focus has to mark elements of the set in a way that picks out something that is in the domain of the question. ${ }^{6}$

\footnotetext{
${ }^{6}$ There is some evidence that attentional focus does not just mark a selection of things but creates a structured "landscape," also deselecting things, possibly arranging them into a hierarchy (Datta
} 


\subsection{Principles of the Erotetic Theory of Attention}

I will now review the crucial elements of the foregoing and use those elements to state the erotetic theory of attention in terms of four terse principles. Attention is a form of sensitivity in the monitoring of tasks. ${ }^{7}$ For the question to arise whether an agent is paying attention to a task, the agent has to be pursuing the task. To pursue a task, the agent actually has to take means toward the goal of her task. I take it that only once one is pursuing a task does the question arise if one is pursuing the task attentively. If an agent uses all of her relevant cognitive resources in a way that will prevent them from serving a task, then the agent is simply not pursuing the task, attentively or otherwise. On the other hand, an agent may pursue a task attentively while making imperfect choices about the cognitive resources to use. Thus, the erotetic theory distinguishes attentive performance from optimal performance.

In section 3.2, I argued that tasks determine completion conditions that can be monitored in the form of structured questions. Again, questions, as they are understood here, are representational contents, not sentences in some language, as described in section 3.1.

(Questions) A task $T$ determines a structured question of the form $\left\langle F_{T}, D_{T}\right\rangle$, with a function $F_{T}$ and a domain $D_{T}$, that encodes $T$ 's completion conditions through its answerhood conditions.

Cognitive systems, be they thought systems or perceptual systems, supply sets of features to the cognitive economy that can encode information relevant to determining

\& DeYoe 2009). Additional formal tools developed in the study of linguistic focus could be adapted for the erotetic theory to model this possibility, but must be left for future work.

${ }^{7}$ Thinking of tasks as the core object of attention is more flexible than it may seem on a first look. We retain a notion of attentional focus as something that corresponds to orientation towards objects, properties, and locations, whose functional role is parasitic on attention to tasks. We can also capture notions like paying attention to a child or to a situation. Intuitively, paying attention to a child means being poised to adopt whatever issues arise from the child. If the child asks a question or makes a request, one makes it one's task to answer the question and fulfill the request, under the constraints of what is reasonable. Paying attention to someone means being responsive to them in a way the fits well with the erotetic theory. Similarly, if one claims to pay attention to a situation, one is ordinarily not saying that one is just accumulating information about it. Paying attention to a situation in the fairly thick intuitive sense means that one is disposed to address those issues or tasks that arise from it as a function of what happens and what one's background commitments are. 
progress in pursuing a task. ${ }^{8}$ We can cognize a task to be completed if we can cognize the question used in monitoring the task as answered by features in our cognitive economy. What can be cognized as an answer to a question in part depends on the assignment of attentional focus, a notion carved out in section 3.3. The functional role of attentional focus is analogous to that of focus in natural language with respect to influencing what can be taken as an answer to a question, as described in section 3.1.

(Focus) Let $A$ be a set of features from any cognitive domain that is available for focus. Let $A_{F O C}$ be the set of those elements of $A$ that are focused.

We can now define what it takes for a set of features contributed by some cognitive system to be a congruent answer to a question.

(Congruent Answerhood) For $\langle F, D\rangle$ a question and $A$ a set of features, $A$ is a congruent answer to $\left\langle F, D>\right.$ if and only if $A_{F O C} \subseteq D \& F\left(A_{F O C}\right) \subseteq A$.

What this means is that if a set of features $A$ is a congruent answer, then what is picked out by focus within $A$ is in the domain of the question (i.e. $A_{F O C}$ is contained in the domain) and actually has the features in the function of the question (i.e. applying the function to what is focused yields something already in $A$ ).

We can now state a condition for attention to a task.

(Attention) An agent pursues a task $T$ attentively to the extent to which the agent is sensitive to the occurrence of congruent answers to $\left\langle F_{T}, D_{T}\right\rangle$, e.g. cognizing the contribution of a process engaged in service of $T$ as an answer if it is a congruent answer to $\left\langle F_{T}, D_{T}>\right.$ and not cognizing it as an answer to $\left\langle F_{T}, D_{T}>\right.$ if it is not a congruent answer to $<F_{T}, D_{T}>$ ?

\footnotetext{
${ }^{8}$ As noted in the previous section, I include feature combinations and positions under the rubric 'feature' to simplify exposition.

${ }^{9}$ To make this fundamentally probabilistic notion precise, one would use a measure of sensitivity like d-prime from signal detection theory that takes into account both hits and false alarms. The present discussion would not obviously benefit from spelling this out, so a more technical reformulation is left for elsewhere. For reasons to make requirements for attention relative to only those processes engaged in service of a task, see Mole (2011).
} 
What this means is that attention is a form of sensitivity in monitoring progress in our tasks. Degrees of attention to a task translate to degrees of sensitivity as a detector for congruent answers to the question implicated in the task. Paying more attention to a task means becoming more sensitive to when a cognitive process one engages in performing the task is producing a congruent answer to the question determined by the task, signaling its completion.

Suppose we are very attentively pursuing the task of finding a banana. The erotetic theory holds that we monitor progress in this task via a question, roughly, "where is there a banana?' As soon as the cognitive systems we are using to perform this task encode the presence of a banana, and our focus is on a position for which the presence of a banana is encoded, we are very likely to cognize the contribution of these cognitive systems as answering the question. In cognizing the question as answered, we take our task to be completed.

To make sense of how the erotetic theory is formulated, it is worth considering nearby alternatives and why they are unattractive. For example, we could make the requirements for attention more restrictive and say that attention demands that we cognize the contribution of any relevant cognitive system as an answer if there is some focus assignment under which it would be an answer to our question. However, this seems too strong. Consider a simple visual search task in which we have to find a red square in a display of red things of various shapes and squares of various colors. This requires serial search, moving our focus to different locations until we find the target. Only once our focus reaches the target can we complete the task, but we do not want to say that we are not attending to the task while we wait for focus to reach the target.

We could also have said that instead of sensitivity to the occurrence of answers in the cognitive economy, more attention demands a higher probability of there being a congruent answer in the cognitive economy. However, consider the task of finding the Fountain of Youth. Hallucination aside, nobody is going to find a congruent answer to the question implicated in this task. However, this does not mean that the task does not impose requirements on attention. You can be blamed by your fellow knights for being distracted in your search in the sense of being in a state in which you would not even recognize an answer if you came across one. 
Finally, instead of taking attention to be a matter of a graded sensitivity to the occurrence of congruent answers, we could have said that we pay attention to a task if and only if whenever something is a congruent answer it will be cognized as an answer. However, this, too, seems too strong. After all, we are dealing with a biological system with noisy processing. Demanding logically perfect detection performance seems likely to make it so we are strictly speaking never paying attention to any task.

\section{Aspects of Attention and Distraction}

I will now consider how the erotetic theory of attention sheds light on various aspects of attention and distraction, contrasting it with the cognitive unison theory where relevant.

\subsection{Attention Demanding Tasks}

The erotetic theory of attention can make sense of why certain tasks make strong demands on attention as we continue to perform them. An example we already discussed was that generating random numbers is very attention demanding (Jahanshahi et al. 2006; Mole 2011).

On the erotetic theory, some tasks may be much more demanding than others in that they decompose into a series of many subtasks. Moving ahead with the broad task constantly requires recognizing that subtasks have been completed. Since moving along with the task requires recognizing regularly that the questions of the subtasks have been answered, one's ability to detect answers is tested on a regular basis and whatever mechanisms underwrite this ability have to constantly be at the ready. For such broad tasks, lower sensitivity to the occurrence of a congruent answer to the question determined by the current subtask (i.e. less attention paid to the task, on the erotetic theory), should normally lower the probability that one can move on to the next subtask and successfully continue pursuing the broad task. Here, a lack of attention quickly leads to not pursuing the broad task at all, since subtasks no longer serve as means to the broad task if we fail to recognize that they have been completed.

On this view, generating random numbers is an attention-demanding task, since we constantly have to detect completion of the subtask of generating a number in one way so we can move on to the subtask of generating the next number a different way, thereby avoiding patterns in our (pseudo-)random sequence. If we fail to recognize that 
we have already completed a given subtask of generating a number in some way, and continue generating more numbers in the same way, we have at some point ceased to generate random numbers in a (pseudo-)random way. Thus, pursuing the task for an extended period of time is virtually impossible without paying attention.

\subsection{Divided Attention and Partial Distraction}

Most people will have experience with the phenomenon that dividing one's attention between multiple tasks is possible and that dividing one's attention can sometimes yield worse performance. Some pairs of tasks can surprisingly be attended to simultaneously without impacting performance, such as sight-reading music while shadowing a text (Allport et al. 1972). Yet, for many pairs of tasks relevant to our daily life, we are not so lucky. Studies suggest that dividing attention between a cell-phone conversation and the task of driving can in fact yield inattentional blindness; we may never become consciously aware of the next pedestrian crossing the road, even if we are looking straight ahead (Scholl et al. 2003).

On the erotetic theory of attention, nothing rules out the possibility of straightforwardly divided attention. Firstly, it is possible to attend to two tasks. We can then ask for each task how sensitive we are to the occurrence of a congruent answer to the corresponding question in the cognitive economy. Depending on what processing capacities are underwriting the relevant detection ability for a given pair of tasks, greater sensitivity for one task may come at the expense of another, or it may not. The erotetic theory allows that attention may be divided unevenly since we can have various degrees of sensitivity for various tasks we pursue simultaneously.

The erotetic theory also suggests when dividing one's attention between two tasks may harm performance. Questions determined by tasks most likely bias how focus is assigned over sets of features contributed by cognitive processes that may be used to perform a task. For example, if we are looking for red things in a task, that may bias our focus toward red things. If cognitive resources for two tasks overlap, biasing attentional focus in service of one task may make it less likely that we find congruent answers for the question of another task. In this case, we might still be attending to both tasks, but it is relatively less likely that one of them will be completed. 
It is useful to contrast this account of the possibility of divided attention with the cognitive unison theory. Mole offers a different view of the relationship between tasks and the cognitive resources one can deploy in service of them. Specifically:

(Cognitive Unison) 'The agent performs [a superordinate task] $\tau$ attentively just in case there is some task $v$ that the agent understands to be a way of performing $\tau$, and just in case the agent is performing $v$ in such a way that the set of cognitive resources that the agent can, with understanding, bring to bear in the service of $v$ does not contain resources that are occupied with activity that does not serve $\tau^{\prime}$ (Mole 2011, p. 65).

Take the (superordinate) task of calculating the sum $2+2$. There are different tasks we can understand to be ways of performing the superordinate task of calculating this sum. Each of these ways of performing the superordinate task requires different sets of cognitive resources. For example, using an abacus requires motor skills, while doing mental arithmetic does not. On the cognitive unison view, we are performing the superordinate task of calculating the sum attentively, just in case we understand that there is a task like using an abacus a certain way or doing mental arithmetic a certain way that is a way of calculating the sum, and the cognitive resources that correspond to one of those ways of calculating the sum are only occupied with the task of calculating the sum.

An acknowledged consequence of the cognitive unison view is the following:

(Non-gradedness) If the relevant resources for a task are occupied with any activity that does not serve the task, there is no attention to the task. Attention is an all-or-nothing phenomenon.

On the cognitive unison theory, attention is an ungraded phenomenon, so there are no genuine cases of partial attention to multiple tasks. In cases like sight-reading music while shadowing text, where there is no interference between tasks, Mole argues that there are two non-overlapping sets of cognitive resources fully devoted to their respective tasks, so we have full attention to two tasks (Mole 2011, p. 74); nothing is being divided. In cases like driving while talking on a cell-phone where there is interference, there is no attention to either task at all, since cognitive resources implicated in the two tasks are not 
exclusively devoted to one task. The only way to account for prima facie cases of unevenly divided or partial attention seems to be to say that they are cases involving intermittent switching from full attention to one task to full attention to another task.

I suggest that it is an advantage of the erotetic theory that it allows for partially divided attention at a time, rather than being forced to postulate momentary switches between full attention to one task and full attention to another. That talking on a cell phone can cause us to fail to notice something unexpected on the road while driving is surprising because our perception of the road seems quite normal to us. Various theories about the role of attention in perception make it implausible to various degrees that no attention is devoted to driving at all, even while we are devoting a large chunk of our attentional resources to following a conversation. It has not been settled how far the role of attention in perception goes, but on many views, at least some involvement of attention appears to be required for normal perception (Treisman 1998; Prinz 2011). If we take any of those views seriously, there is pressure on the cognitive unison theory to say that attention 'flickers' back and forth between the tasks to make sense of the fact that perception seems normal. This would lead one to expect some form of perceptual flickering that does not obviously obtain. Of course, the relevant flickering could be happening so fast that we simply do not notice any perceptual effects. Yet, absent strong reasons to believe that there is attentional flickering in all cases of divided attention involving performance costs, it seems to be an advantage for the erotetic theory to be able to account for these cases without having to rely on flickering attention.

\subsection{Attentional Focus, Content, and Phenomenology}

It is widely recognized that attentional focus can change phenomenal character (or what our experience is like) even as gaze and stimulus remain fixed (Peacocke 1993; Chalmers 2004; Block 2010). Maintain ocular fixation on the leftmost $\mathrm{T}$ in (13) and shift attentional focus between the gray and black Ts.

$\begin{array}{lllll}\mathbf{T} & \mathrm{T} & \mathbf{T} & \mathrm{T} & \mathbf{T}\end{array}$

The character of our perceptual experience changes as we shift our attentional focus in this way. One attempt to capture this phenomenal change would be to say that as we are 
shifting our attentional focus, we are representing either the gray Ts or the black Ts as more salient (Chalmers 2004). There is an ongoing debate about how to account for shifts in phenomenal character induced by shifts in attentional focus and whether this can ultimately be done in representationalist terms (Block 2010).

The erotetic theory of attention opens up new avenues for characterizing the impact of attentional focus on phenomenal character in representationalist terms. Consider the proposal that attentional focus contributes a representation of saliency to phenomenal character. For a certain kind of representationalist, it would be attractive to be able to fulfill the following desiderata in giving an account of saliency:

1. We are not representing properties of external objects that do not objectively exist.

2. We are not representing our own mental states in representing something as salient. Experience remains 'transparent' (Harman 1990).

3. Since salience is a representational content, it has non-trivial accuracy conditions (see Siegel 2010).

For example, if we take salience to be a property of external objects like shape, we will have to conclude that we always misrepresent when we take it that some things have this property while others do not, since there is no corresponding asymmetry in the distal layout. If we take it that salience is a property of our own mental representations reflected in phenomenal character, we can no longer claim that our perceptual phenomenology is in all its aspects transparent onto the external world.

The erotetic theory of attention allows us to propose a representational content to correspond to salience that satisfies our three desiderata. We can say that the phenomenology of salience supervenes on an independently motivated representational contribution of focus. Focus represents things as corresponding to the domains of our questions. Those questions themselves fundamentally correspond to sets of alternative situations; questions are contents not mental states. If what is picked out by focus does not match any of our questions, focus momentarily misrepresents. Thus, the erotetic theory of attention can give us accuracy conditions for salience without having to insist that we represent perceived distal properties as changing with changes in what we perceive as salient or having to insist that saliency represents mental states. Moreover, the 
relevant representational content in fact plays a crucial role in the 'gear box' of attention and is not just stipulated to defend representationalism. The erotetic theory of attention does not force us to give this account of salience, since claims about what phenomenal character supervenes on are independent from the core theory. However, by making this view available, the erotetic theory of attention creates a new avenue for a certain kind of representationalist that seems intuitively appealing.

\subsection{Distraction and Practical Rationality}

I will now suggest that the erotetic theory of attention sheds new light on distraction, the 'flipside' of attention. Begin with a simple example scenario involving distraction. ${ }^{10}$

(Buzzy and the scholar) Jack is a dedicated scholar trying to finish a difficult chapter in a book. The room is completely silent. Suddenly a fly begins buzzing. At first, Jack is merely annoyed but manages to keep working. After a while, Jack decides he has to deal with the fly before he can continue.

In this scenario, the fly is a distractor. There is an intuitive sense in which one can initially resist a distractor of this sort and continue to attend to one's task, though performance may already suffer to some extent.

How do distractors relate to attention? A buzzing fly against the background of a silent room will intermittently attract focus because of its high contrast and relative novelty compared to other stimuli, through mechanisms largely independent of conscious decision-making (see section 3.3 on focus). However, focus on the fly is not going to yield anything congruent to the question involved in the scholar's task. Here, it is time to link the proposed account of attention to the question of motivation. I suggest that when focus yields something incongruent to the question implicated in one's task, the result is a degree of a very specific kind of psychological pain or frustration. Call that specific kind of pain 'incongruency pain'. By contrast, when we get a congruent answer to the question implicated in our task, the result is a degree of a very specific kind of psychological pleasure. We can imagine that there is an account balance to which the relevant specific

\footnotetext{
${ }^{10}$ Mole discusses a similar example involving a fly as a distractor in Mole (2011, p. 47) though his concern is not with the dynamics of resisting distraction.
} 
kind of pleasure adds and from which the relevant specific kind of pain or frustration subtracts. Call that the agent's 'congruency balance'. If the congruency balance reaches a certain tolerance threshold, the system will take the most direct available means to avoiding further subtraction. A special limit on our ability to will ourselves to tolerate the relevant specific kind of incongruency pain (really a kind of task-related frustration) might be highly adaptive for the sorts of creatures we are, especially if we consider that human nature is hardly optimized for office work or scholarly work, as Kant already seemed to recognize (Zammito, 2001, p. 407 citing Metaphysik Herder, 28.2:881). ${ }^{11}$ The foregoing remarks have to remain tentative, but they will help show how the erotetic theory of attention can help connect issues about attention with agency.

With this proposal, we can make sense of the intuitively compelling idea that we can resist the fly as a distractor. The fly attracts focus in a way that will make it very likely that focus yields something incongruent to the task Jack is engaged in. This yields subtraction from his congruency balance. Jack can resist and wait for his focus to return to where he needs it to be to continue making progress on his chosen task. Once Jack's tolerance threshold is reached, he cannot do otherwise but take the most direct available means to avoiding further incongruency. The most direct means is to change the task to locating the fly for the purposes of silencing it. That way, focus on the fly immediately produces a congruent answer (Jack has adopted a new task designed to be congruent to whatever focus is providing) and the congruency balance can increase again. However, the fly has now succeeded in distracting Jack from his task by supplanting the task Jack originally decided to pursue.

I will now argue that it is a virtue of this analysis of distractors that it can shed some light on when it makes sense to identify distractibility as a form of practical irrationality. Consider Korsgaard's well-known scenario intended to illustrate a form of practical irrationality.

\footnotetext{
${ }^{11}$ It might make evolutionary sense to have a reward system that will prevent us from indefinitely sticking with tasks that are never completed. If searching for a banana never yields a banana, we might be well-advised to search for something else, long before winter comes and we feel pain from hunger. It might help for such a reward system to have some independence from generic pleasure and pain. Prolonged fruitless search that is intrinsically pleasurable could still be dangerous in keeping us from searching for alternatives before it is too late. Similarly, a painful but well-progressing chase is not automatically frustrating. It might make sense to think of multiple pleasure/pain, congruency/incongruency balances with 'exchange rates' that depend on context.
} 
(Jeremy) 'Jeremy settles down at his desk one evening to study for an examination.

Finding himself a little too restless to concentrate, he decides to take a walk in the fresh air. His walk takes him past a nearby bookstore, where the sight of an enticing title draws him in to look at a book. Before he finds it, however, he meets his friend Neil, who invites him to join some of the other kids at the bar next door for a beer. Jeremy decides he can afford to have just one, and goes with Neil to the bar. When he arrives there, however, he finds that the noise gives him a headache, and he decides to return home without having a beer. He is now, however, in too much pain to study. So Jeremy doesn't study for his examination, hardly gets a walk, doesn't buy a book, and doesn't drink a beer.' (Korsgaard 2008, p.59 n. 52)

Korsgaard goes on to suggest that there is a problem with Jeremy as an agent, because what he does at any given moment is close to an accident. Jeremy's actions are uncomfortably close to mere reactions to the environment.

Take Jeremy from the point at which he is going for a walk. Let's say he is really going for a walk to buy a newspaper, to make clear that he starts out with a determinate task. His focus is drawn-in by a book, a friend, and a beer in short succession. The book, the friend, and the beer attract his focus, regardless of his task of buying a newspaper. Yet, those things his focus is drawn to are incongruent to the question implicated in his task. In yielding incongruity, the book, the friend, and the beer produce a specific kind of psychological pain subtracting from his congruency balance. Each time one of these distractors appears, Jeremy has two options to mitigate the loss to his congruency balance. He can wait for his focus to shift again, fickle as it is, or he can change his task to make it congruent with whatever his focus is drawn to. Here, Jeremy needs to make a choice.

It seems justified to call Jeremy practically irrational if his tolerance threshold for incongruency is not yet reached but he responds to the pain of incongruency by just letting his task be dictated by whatever distractor is momentarily impinging on him. In this case, Jeremy is practically irrational because he is not using all available cognitive means to pursue his chosen task (stipulate that the book, friend, etc. are not compelling reasons to abandon his task). At the same time, we can allow that there could be someone 
with an extremely low incongruency tolerance threshold who would exhibit the same behavior as Jeremy without being practically irrational. This individual simply could not do otherwise, making him unfortunate but not practically irrational as an agent.

In sum, the erotetic theory of attention opens up a way of distinguishing irrational use of one's attentional resources, however limited or extensive they may be, from limitations of how extensive those resources are. People suffering from certain deficits may not come out as irrational even if they behave like Jeremy.

\section{Conclusion}

According to the erotetic theory of attention presented in this paper, attention is a matter of the relationship between questions (encoding the completion conditions for tasks for the purposes of monitoring) and what counts as answers to those questions. This relationship was made precise using formal tools developed in the semantics of natural language. Yet, nothing in the account requires language as a precursor to attention. A number of attractive features of the erotetic theory were discussed. It allows for divided attention and allows that attention comes in degrees. The theory also sheds some light on issues concerning the relationship between attention, representational content, and phenomenology that have recently been debated. Moreover, the theory sheds some light on distraction, and of when being distractible suggests a defect in practical rationality.

\section{Philosophy-Neuroscience-Psychology Program \\ Washington University in St. Louis}

\section{References}

Allport, D. A. 1987: Selection for action: Some behavioral and neurophysiological considerations of attention and action. In Heuer, H. and Sanders, A. F., (eds.) Perspectives on Perception and Action. Lawrence Erlbaum.

Allport, D. A., Antonis, B., and Reynolds, P. 1972: On the division of attention: a disproof of the single channel hypothesis. Quarterly Journal of Experimental Psychology, 24(1), 22535 .

Block, N. 2010: Attention and mental paint. Philosophical Issues, 20, 23-63.

Botvinick, M., Braver, T., Barch, D. Carter, C. \& Cohen, J. 2001: Conflict monitoring and cognitive control. Psychological Review, 108(3), 624-652.

Burge, T. 2009: Perceptual objectivity. Philosophical Review, 118 (3), 285-324.

Campbell, J. 2002: Reference and Consciousness. Oxford: Oxford University Press. 
Chalmers, D. 2004: The representational character of experience. In B. Leiter (ed), The Future for Philosophy. Oxford: Oxford University Press.

Datta, R. and DeYoe, E. A. 2009: I know where you are secretly attending! The topography of human visual attention revealed with fMRI. Vision Research, 49(10), 1037-44

Desimone, R. 2009: Neural synchrony and selective attention. Neural Networks, IJCNN 2009, IEEE, 683-684.

Desimone, R., and Duncan, J. 1995. Neural mechanisms of selective visual attention. Annu. Rev. Neurosci, 18, 193-222.

Dwyer, D. 2007: Husserl's appropriation of the psychological concepts of apperception and atten tion. Husserl Studies, 23(2), 83-118.

Eilan, N., Hoerl, C., McCormack, T., Roessler, J. (eds) 2005: Joint Attention: Communication and Other Minds : Issues in Philosophy and Psychology. Oxford: Clarendon Press.

Groenendijk, J. and Stokhof, M. 1984: Studies on the Semantics of Questions and the Pragmatics of answers. Ph.D. dissertation, University of Amsterdam.

Hamblin, C. L. 1958: Questions. The Australasian Journal of Philosophy, 36, 159-168.

Harman, G. 1990: The intrinsic quality of experience, Philosophical Perspectives, 4, 31-52.

Itti, L., and Baldi, P. 2005: A principled approach to detecting surprising events in video. Pro ceedings in IEEE Conference on Computer Vision and Pattern Recognition (CVPR), 631-637.

Itti, L. and Baldi, P. 2009: Bayesian surprise attracts human attention. Vision Research, 49, 1295-1306.

Jahanshahi, M., Saleem, T., Ho, A. K., Dirnberger, G., and Fuller, R. 2006: Random number generation as an index of controlled processing. Neuropsychology, 20(4), 391-99.

Jiang, Y., Costello, P., Fang, F., Huang, M., and He, S. 2006: A gender- and sexual orientationdependent spatial attentional effect of invisible images. PNAS, vol. 103(45), 1704817052.

Kastner, S. and Ungerleider, L. G. 2000: Mechanisms of visual attention in the human cortex. Annu. Rev. Neurosci, 23, 315-341.

Krifka, M. 2001: For a structured account of questions and answers. In: C. Féry and W. Sterne feld (eds). Audiatur vox sapientiae. A Festschrift for Achim von Stechow, Berlin: Akade mie-Verlag, 287-319.

Korsgaard, C. 2008/1997: The normativity of instrumental reason. Reprinted in: C. Korsgaard. The Constitution of Agency: Essays on Practical Reason and Moral Psychology. Oxford: Oxford University Press, 27-69.

McNevin, N. H., Shea, C. H., and Wulf, G. 2003: Increasing the distance of an external focus of attention enhances learning. Psychological Research, 67, 22-29.

Mill, J. S. 1865/1979: An Examination of William Hamilton's Philosophy and of the Principal Philosophical Questions Discussed in his Writings. In: J. M. Robson (ed) The Collected Works of John Stuart Mill, Vol. IX, London: Routledge.

Mole, C. 2011: Attention is Cognitive Unison: An Essay in Philosophical Psychology. Oxford: Oxford University Press.

Peacocke, C. 1993: Review of M. Tye, The imagery debate. Philosophy of Science, 60, 675-677.

Prinz, J. 2011: Is attention necessary or sufficient for consciousness? In: C. Mole, D. Smithies, and W. Wu (eds) Attention: Philosophical and Psychological Essays. Oxford University Press.

Reynolds, J., and Desimone, R. 2000: Competitive mechanisms subserve selective visual atten tion. In: Marantz, A. Miyashita, Y., O’Neil, W. (eds). Image, Language, Brain. Cam bridge: MIT Press, 233-47.

Reynolds, J. H., and Heeger, D. J. 2009: The normalization model of attention. Neuron, 61, 168185.

Scholl, B. J., Noles, N. S., Pasheva, V., \& Sussman, R. 2003: Talking on a cellular telephone dramatically increases sustained inattentional blindness. Journal of Vision, 3:9(156a).

Siegel, S. 2010: The contents of perception. Stanford Encyclopedia of Philosophy, Accessed 
May 2012.

Smithies, D. 2011: Attention is rational-access consciousness. In Mole, C., Smithies, D., and Wu, W., (eds.). Attention: Philosophical and Psychological Essays. Oxford University Press.

Treisman, A. and Gelade, G. 1980: A feature integration theory of attention. Cognitive Psychol ogy, 12, 97-136

Treisman, A. 1998: The perception of features and objects. In: A. Baddeley, and L. Weiskrantz (eds.). Attention: Selection, Awareness and Control. New York: Oxford University Press, 5-35.

Watzl, S. 2011: Attention as structuring the stream of consciousness. In Mole, C., Smithies, D., and $\mathrm{Wu}, \mathrm{W} .,($ eds.). Attention: Philosophical and Psychological Essays. Oxford University Press.

Wright, R. D., and Ward, L. M. 2008: Orienting of attention. Oxford: Oxford University Press.

Wolfe, J. M. 2000: Attention is fast but volition is slow. Nature, vol. 406, 691.

Wu, W. 2011: Confronting many-many problems: Attention and agentive control. Nous, $45(1), 50-76$.

Wulf, G., and Prinz, W. 2001: Directing attention to movement effects enhances learning: A review. Psychonomic Bulletin and Review, 8, 648-660.

Zammito, J. 2001: Kant in the 1760s: Conceptualizing the 'popular turn'. In: P. Cicovacki (ed.) Kant's Legacy: Essays in Honor of Lewis White Beck, Rochester: University of Rochester Press, 387-433. 